\title{
Interactive comment on "Hygroscopic properties and CCN activity of atmospheric aerosols under the influences of Asian continental outflow and new particle formation at a coastal site in East Asia” by Hing Cho Cheung et al.
}

Hing Cho Cheung et al.

ckchou@rcec.sinica.edu.tw

Received and published: 22 January 2020

Thank you for the valuable comments. We have further revised our manuscript according to the comments.

Major and General Comments: 1) Page 5 line 17: The measurement intervals are described. The CCN counter need several minutes after supersaturation changes to stabilize. It is of high importance to remove these time periods from the dataset. It is unclear if this correction has been done for the current analysis. This correction is 
absolutely needed.

Ans: For CCN measurement, only last 5 mins data were used to represent the NCCN at different SS settings, and in kappa calculation as well. This measure was mentioned in text.

Page 5, line 22. "Since CCNC needs several minutes to stable after changing the SS setting, therefore only the last 5 minutes data were used in kappa calculation." has been added.

2) Page 5 line 20-21: The authors mention that the CCNC was calibrated, was the CCN calibrated according to Rose et al. (2008)? If not this needs to be further explained. If yes the manuscript needs to be cited. Was the data corrected of the maximal activated fraction, which is of high importance in particular for total CCN measurements (Paramonov et al., 2013; Rose et al., 2010)? In addition, it would be good to refer to a manuscript which describe operation of the DMT CCNC (e.g. Lance et al., 2006).

Ans: The CCNC was not calibrated exactly according to Rose et al. (2008) and the methodology was further explained. The data was corrected of the maximal activated fraction according to Rose et al. 2010.

Page 5, line 29. The following sentences have been added:

"Since the counting efficiencies of CCNC were lower than CPC, thus the maximum activated fraction of NCCN/NCN would be smaller than 1. Therefore, the activation diameters used to calculate the SS values were determined by using half of maximum activated fraction of NCCN/NCN (Rose et al. 2010). The operation of the DMT CCNC adopted in this study can be referred to Lance et al., 2006."

3) Page 6 line 13-14: The hygroscopicity parameter kappa is very sensible and can be easily calculated wrong using equation 1 . The common way to calculate kappa by total CCN number concentration by size-resolved CCN measurements (e.g. Rose et al. 2010; Irwin 2011; Gunthe et al. 2011; Jurányi et al., 2011; Pöhlker et al., 2016). 
Equation 1 can be used if the CPC and the integral of SMPS is the same for the whole measurement period, however, in this case the denominator of equation 1 will be cut out.

Ans: The kappa values were recalculated by using the equation (6) proposed by Petters and Kreidenweis, with the apparent cut-off diameter of CCN activation (Dcut) which is diameter above the integral NCN equals the observed NCCN. The correspond kappa values (Kcut) was suggested to represent effective average hygroscopicity of CCNactive particles in the size range above Dcut (Rose et al. 2010).

The equation (1) in manuscript has been replaced by equation (6) in Petters ad Kreidenweis (2007). The description about the kappa calculation has been revised in Section 2.2 in Page 6.

4) Page 8 line 2 and Table 2: The values for kappa reported in this study are different to former studies; this is also described by the author. It should be discussed if the different to other studies is coast by the method which has been used and if yes the analysis should be redone.

Ans: More discussion about the difference between the present study to other coastal studies has been added.

Page 8, line 28. "The larger variations of $\kappa$ values obtained at CAFÉ station compared to the coastal studies at Hong Kong and Noto Peninsula Japan (Meng et al., 2014, Iwamoto et al., 2016) may attribute to the shorter periods of measurements in these two studies which lasted for nearly 1 month in May and October, respectively, while the present study lasted for 1 year. Moreover, the $\kappa$ values reported in these previous Asian studies were derived by size-resolved $\mathrm{CCN}$ data which represent the average hygroscopicity of he activated aerosol around the activation diameter (Da), while the kappa was calculated by Dcut in this study which represent the average hygroscopic of the aerosol above the size of Dcut. Nevertheless, the aerosol composition at CAFÉ station were frequently influenced by local pollution from urban region and regional 
pollution associated with winter monsoon through different seasons, as indicated in previous studies (Chou et al., 200, 2010, 2017), hence this explains the larger variations in $\kappa$ values observed in this study. The influence of different airmass on aerosol hygroscopicity will be discussed in Section 3.2."

5) Page 8 line 5: By comparing the calculated kappa to former studies, the difference in the methods to calculate kappa needs to be discussed. Meng et al. (2014) and Iwamoto et al. (2016) measured size resolved CCN properties and Schmale et al. (2018) calculated by using AMS measurements. These findings should also be compared to Irwin et al (2011).

Ans: The difference of kappa calculation between the size-resolved CCN from previous studies, and the integral CCN from this study has been mentioned.

Please refer to comment \#4.

6) Page 10 line 25 and Figure 6: The author describes new particle formation events (NPF) in figure 6. I cannot see a significant nucleation mode in Figure 6 in the NPF case. The NPF case seems more like an Aitken mode aerosol size distribution while the non-NPF case is a bimodal Aitken and accumulation mode aerosol size distribution. Also the fitting is not convincing.

Ans: The multiple log-normal fitting for the particle size distribution data in this study was calculated by the DO-FIT method which widely applied in a number of previous NPF studies. There is larger variance of nucleation mode particle in NPF group, and the GMD was within nucleation mode. It is plausible that the concentration of the nucleation mode particles was not significantly high, but the PSD clearly indicated that a NPF process was undertaken at CAFÉ station (see Figure 7). Also, the Aitken mode particle $(\sim 100 \mathrm{~nm})$ always existed at CAFÉ station, therefore, the particles with diameter near 100nm were both observed in NPF and non-NPF groups.

7) Page 11 line 5-7: The number concentration of particles smaller $30 \mathrm{~nm}$ is reported. 
How was this number calculated? It can be quite difficult to measure particles smaller $10 \mathrm{~nm}$. It would be important to know the lower cut off for particles smaller $30 \mathrm{~nm}$.

Ans: The N30 was the number concentration between $13.6 \mathrm{~nm}<=\mathrm{d}<=30 \mathrm{~nm}$, and N30100 was the number concentration between $30 \mathrm{~nm}<d<=100 \mathrm{~nm}$ as well as $\mathrm{N100-736}$ $(100 \mathrm{~nm}<\mathrm{d}<=736 \mathrm{~nm})$. The description about the terms N30, N30-100 and N100-736 has been added in Section 2.

Page 5, line 11. "The nucleation, Aitken and accumulation modes particle number concentrations were represented by N30 (13 nm < d <= 30 nm), N30-100 (30 nm < d $<=100 \mathrm{~nm})$ and N100-736 $(100 \mathrm{~nm}<\mathrm{d}<=736 \mathrm{~nm})$., respectively."

8) Page 11 line 25: The authors report that the increase in CCN cannot be explained by the growth of NPF because the additional CCN were observed at an initial stage of form does not indicate a NPF in an initial state. This is, however, very difficult to detect and need special care in the inlet system. The findings of the manuscript leads to summery that growth of newly formed particle can explain the increase in CCN.

Ans: We agreed that it is very difficult to measure the nucleation-mode particles of very small size, and in this study our smallest size of measurement is $13 \mathrm{~nm}$. However, an significant CCN increased has been observed accompanying the growth of particles at the later stage of NPF process (see Figure 7), but it is less conclusive to link the increased of CCN to the initial growth of newly formed particles. Hence, the discussions relevant to this has been removed and this section has been rewritten.

9) Page 12 line 33-34: This sentence is not clear. The sentence needs to be restructured.

Ans: This section has been rewritten.

Printer-friendly version

10) Overall the discussion and the final finding is very much focusing on the NPF. This is not well described in the method section and the NPF events are not clearly shown.

I would recommend to concentrate on the CCN key parameters in the discussion and 
do not focus on NPF. If the author is willing to stick to the NPF discussion the method section need to include the losses in the sampling, the well calibrated detection limit of the instruments and the NPF events need to be presented very clearly and convincing in the manuscript.

Ans: Agreed. We have rewritten this section in the manuscript. Please see previous reply to comment \#8.

Interactive

comment

Minor Comments 11) The abstract would benefited from representative values for kappa, NCCN and NCN.

Ans: The abstract has been revised to include the values of kappa, NCCN and NCN.

Abstract: The chemical composition of fine particulate matters (PM2.5), the size distribution and number concentration of aerosol particles (NCN) and the number concentration of cloud condensation nuclei (NCCN) were measured at the northern tipo of Taiwan Island during a continuous measurement from April 2017 to March 2018. The parameters of aerosol hygroscopicity (i.e. activation ratio, activation diameter and kappa) were retrieved from the measurements. Significant variations were found in the hygroscopicity of aerosols ( $\kappa: 0.18-0.56$, SS: $0.12-0.80 \%$ ), which were subjected to various local and regional pollution sources, including aged air pollutants originating in the eastern/northern China and transported on the Asian continental outflows, fresh particles emitted from local sources and distributed by land-sea breeze circulations as well as produced by new particle formation (NPF) processes. Cluster analysis was applied to the backward trajectories of air masses to investigate their respective source regions. The results showed that the aerosols associated with Asian continental outflows were characterized with lower NCN, NCCN and higher kappa values (NCN: 2520 $\mathrm{cm}-3, \mathrm{NCCN}: 1110 \mathrm{~cm}-3$ and $\kappa: 0.42$ at SS: 0.28\%), whereas higher NCCN and NCN with slightly lower kappa values (NCN: $4850 \mathrm{~cm}-3$, NCCN: $1460 \mathrm{~cm}-3$ and $\kappa: 0.40$ at SS: $0.28 \%$ ) were found for aerosols in local air masses.

Printer-friendly version

12) Page 4 line 20: it would be helpful if the author could cite a reference for the station. 
Ans: A reference for the CAFÉ station has been added in Section 2. Page 4, line 26. "More detailed information of the site description of CAFÉ station can be referred to Chou et al. 2017."

13) Page 8 line 16: "It is noteworthy that both the $\kappa$ and DSS decrease with the SS". Even if kappa would stay the same Dss would still decrease with SS. And when kappa decreases for a given SS, Dss is increasing not decreasing in comparison relative to the Dss of the same kappa.

Ans: We agreed with the comment, but the fact that kappa decrease for a given SS and DSS should be increasing applies if large and small particles have same hygroscopicity. Our observation showed that an increase in SS caused a decrease in $\mathrm{k}$ and Dss (Dcut). We suggested that in our case, when SS increases, smaller and less hygroscopic particles present get larger and is considered as the activated particles. As the cut-off diameter (Dcut) was adopted (Rose et al. 2008), a smaller k was computed.

Page 9, line 8: To better clarify, the text has been revised as "It is noteworthy that both the $\kappa$ and Dcut were observed with the increase of SS. This could due to the difference in the chemical composition of the aerosols for smaller and larger particles at the site, with less hygroscopic species in smaller particles and more hygroscopic species in larger particles. As the SS increases, smaller and less hygroscopic particles present get larger and is considered as the activated particles. As the cut-off diameter was adopted (Rose et al., 2008), a smaller $\kappa$ was computed."

14) Figure 7: The axes of the particle size distribution have only one number this make it impossible to interpret the figure.

Ans: Noted. Figure 7 has been re-plotted.

15) Figure 8: The condensation vapors should be located left of the NPF. The process described in the figure is not the only way to increase CCN by NPF. The condensation vapors for example would usually first condense on bigger particles. Following I would

Printer-friendly version

Discussion paper
Interactive

comment 
not show this figure in the pressed form as a final finding in the manuscript.

Ans: We agreed on this. The observed increase CCN could be due to other processes as well, and it is not conclusive to attribute this only to NPF in this study. This could due to the fact that the smallest particle size measured in this study is around $13 \mathrm{~nm}$, and hence we cannot conclude on the increase in CCN from the newly formed particles at the early stage of NPF. Nevertheless, a significant increase in CCN has been observed with the growth of particles at the later stage of NPF process (see Figure 7). The discussion has been revised accordingly.

Page 12, line 9. The sentences have been revised as "Nevertheless, the observed increased in CCN accompanying with the growth of particles could due to various mechanisms (e.g. vapor condensed on existing sub-CCN, coagulation between CCNs, and other oxidation process), and the cause to the increase in CCN and its relation to NPF needs to be further studies." Figure 8 has been omitted in the manuscript.

Interactive comment on Atmos. Chem. Phys. Discuss., https://doi.org/10.5194/acp-2019-519, 2019. 